\title{
Surgery followed by concurrent radiochemotherapy as treatment for patients with locally recurrent cervical cancer
}

\author{
Xiang Zhang ${ }^{1,2}$, Zhongbo Chen ${ }^{3}$, Jianhong Chen ${ }^{1,2}$, Junjian Wang $^{3}$, Yingchang Wang ${ }^{1,2}$, Jianqing Zhu ${ }^{3}$ \\ ${ }^{1}$ Department of Gynecologic Radiation Oncology, Cancer Hospital of the University of Chinese Academy of Sciences (Zhejiang Cancer Hospital), \\ Institute of Cancer and Casic Medicine (IBMC), Chinese Academy of Science, Hangzhou, China; ${ }^{2}$ Zhejiang Key Laboratory of Radiation Oncology, \\ Hangzhou, China; ${ }^{3}$ Department of Gynecologic Surgery, Cancer Hospital of the University of Chinese Academy of Sciences (Zhejiang Cancer \\ Hospital), Institute of Cancer and Casic Medicine (IBMC), Chinese Academy of Science, Hangzhou, China \\ Contributions: (I) Conception and design: X Zhang; (II) Administrative support: J Zhu; (III) Provision of study materials or patients: Z Chen, J Wang; \\ (IV) Collection and assembly of data: J Chen, Y Wang; (V) Data analysis and interpretation: X Zhang; (VI) Manuscript writing: All authors; (VII) \\ Final approval of manuscript: All authors. \\ Correspondence to: Jianqing Zhu. Department of Gynecologic Surgery, Zhejiang Cancer Hospital, No.1, East Banshan Road, Gongshu District, \\ Hangzhou 310022, China. Email: zhujq@zjcc.org.cn.
}

Background: No standard treatment exists for patients with recurrent cervical cancer. This study aimed to determine the role of surgery, followed by concurrent radiochemotherapy, as a treatment for recurrent pelvic cervical cancer without previous radiotherapy.

Methods: The current study identified patients diagnosed with vaginal and/or pelvic sidewall recurrent cervical cancer after primary surgery without radiotherapy in Zhejiang Cancer Hospital from May 2012 to November 2016. These enrolled patients underwent surgery, followed by concurrent radiochemotherapy and data were analyzed.

Results: This study enrolled 27 patients, including 11 with central (vaginal) and 16 with noncentral (pelvic sidewall with or without vaginal) recurrences. The median follow-up time after recurrence was 64 months (range, 5-110 months). All patients underwent surgery to resect the tumor as completely as possible and pelvic external beam radiotherapy (EBRT) with a median equivalent dose in 2 Gy fractions (EQD2) of 45.1 Gy (range, 44.3-47.8 Gy) with concurrent weekly cisplatin chemotherapy. The median EQD2 dose for the tumor bed/residual tumor was 51 Gy (range, 44.3-73.4 Gy), including 18 patients with a boost dose by EBRT or vaginal brachytherapy (VBT). The 5-year overall survival (OS) rates were $77.1 \%$ (central) and $65.7 \%$ (noncentral) without a statistically significant difference $(\mathrm{P}=0.246)$. Progression-free survival (PFS) rates were $81.8 \%$ (central) and $34.4 \%$ (noncentral), respectively, with a statistically significant difference $(\mathrm{P}=0.047)$. Three patients with noncentral recurrence experienced grade $\geq 3$ complications associated with surgery.

Conclusions: Surgery followed by concurrent radiochemotherapy was a feasible and effective treatment with acceptable complications for locally recurrent cervical cancer, which markedly improved the survival of pelvic sidewall recurrence.

Keywords: Cervical cancer; recurrent; surgery; radiochemotherapy

Submitted Jul 03, 2021. Accepted for publication Aug 25, 2021.

doi: $10.21037 /$ tcr-21-1163

View this article at: https://dx.doi.org/10.21037/tcr-21-1163 


\section{Introduction}

Cervical cancer remains the most common gynecological cancer worldwide, especially in developing countries (1). Although the proportion of early-stage patients has increased, a recurrence rate of $11-22 \%$ has occurred in the International Federation of Gynecology and Obstetrics stages I-II patients after primary surgery (2-4). Hence, curing these recurrent patients is challenging.

There is no standard treatment for recurrent cervical cancer patients. Traditionally, radiotherapy with or without chemotherapy is preferable for recurrent patients undergoing primary surgery without radiotherapy (both central and noncentral pelvic recurrence). However, the survival of noncentral pelvic recurrence is poor, with a 5 -year overall survival (OS) rate range from $14 \%$ to $41.82 \%(5-11)$. Poor survival may be due to the inadequate radiation dose delivered to the recurrent lesion, restricted by the normal organs close to the lesion. The dose delivered to the noncentral tumor is generally a maximum of $65 \mathrm{~Gy}$, below the theoretical lethal dose. Several studies enrolled a small number of recurrent patients undergoing surgery when reporting a series of recurrent patients, but the role of surgery was not specifically studied. However, surgery is a potentially promising treatment for these patients because the prerequisite radiation dose for the tumor bed or residual tumor could be lower after surgery. From the National Comprehensive Cancer Network (NCCN) guideline, a description was noted on "consider surgical resection if feasible" and then "individualized external beam radiotherapy $(\mathrm{EBRT}) \pm$ systemic therapy \pm brachytherapy (BT)" for local/regional recurrence. However, the guideline does not cite any references. This study focused on the role of surgery, followed by concurrent radiochemotherapy, as a treatment for recurrent pelvic cervical cancer without previous radiotherapy. We present the following article in accordance with the STROBE reporting checklist (available at https://dx.doi.org/10.21037/tcr-21-1163).

\section{Methods}

\section{Patients}

The current study identified patients diagnosed with recurrent cervical cancer after primary surgery without previous radiotherapy in Zhejiang Cancer Hospital from May 2012 to November 2016. All the recurrences were confirmed by histopathology. Patients with distant and lymph node metastases were excluded. The recurrent sites were the vagina, pelvic sidewall, or both. Central recurrence was defined as vaginal recurrence and noncentral recurrence was defined as pelvic sidewall recurrence with or without vaginal recurrence. These enrolled patients underwent surgery followed by radiotherapy with or without chemotherapy. The study was conducted following the Declaration of Helsinki (as 2013 revision). The Ethics Committee of Zhejiang Cancer Hospital approved this study (No. 2015-01-99), registered with the following number: ChiCTR1800019960 and individual consent for this retrospective analysis was waived.

\section{Treatment decision}

The treatment is decided based on pelvic examination, imaging data including computed tomography, magnetic resonance imaging, and positron emission tomographycomputed tomography and patient opinion. The patient was advised to undergo surgery and postoperative radiotherapy if distant metastasis was ruled out and no serious surgical complications were expected. The same group of doctors, composed of gynecologic oncology surgeons and gynecologic radiation oncologists, treated all patients.

\section{Surgery}

The principle of surgery is to resect the tumor as completely as possible, but a safety margin was not indispensable to avoid severe complications. In most cases, en bloc resection of the recurrent tumor was performed during the operation. In some situations, complete resection was not possible, for example, when tumors surrounded the vessels. Thus, tumor cytoreductive surgery was performed. Although the visible residual tumor was burned electrically, these cases were defined as residual tumor cases for positive surgical margin. Patients with partial cystectomy/ureterectomy or ureter/ bladder injury underwent ureteral stent placement.

\section{Radiotherapy and chemotherapy}

All patients underwent pelvic radiotherapy, including the upper vagina, after surgery with a dose of at least 45 Gy. Inguinal radiation was performed if the lower vagina was involved. Delineation of clinical target volume followed the Radiation Therapy Oncology Group recommendation. A 7-mm margin was used for planning target volume and a $15-20-\mathrm{mm}$ margin for vaginal cuff movement. Some patients received a boost dose for tumor bed or residual 


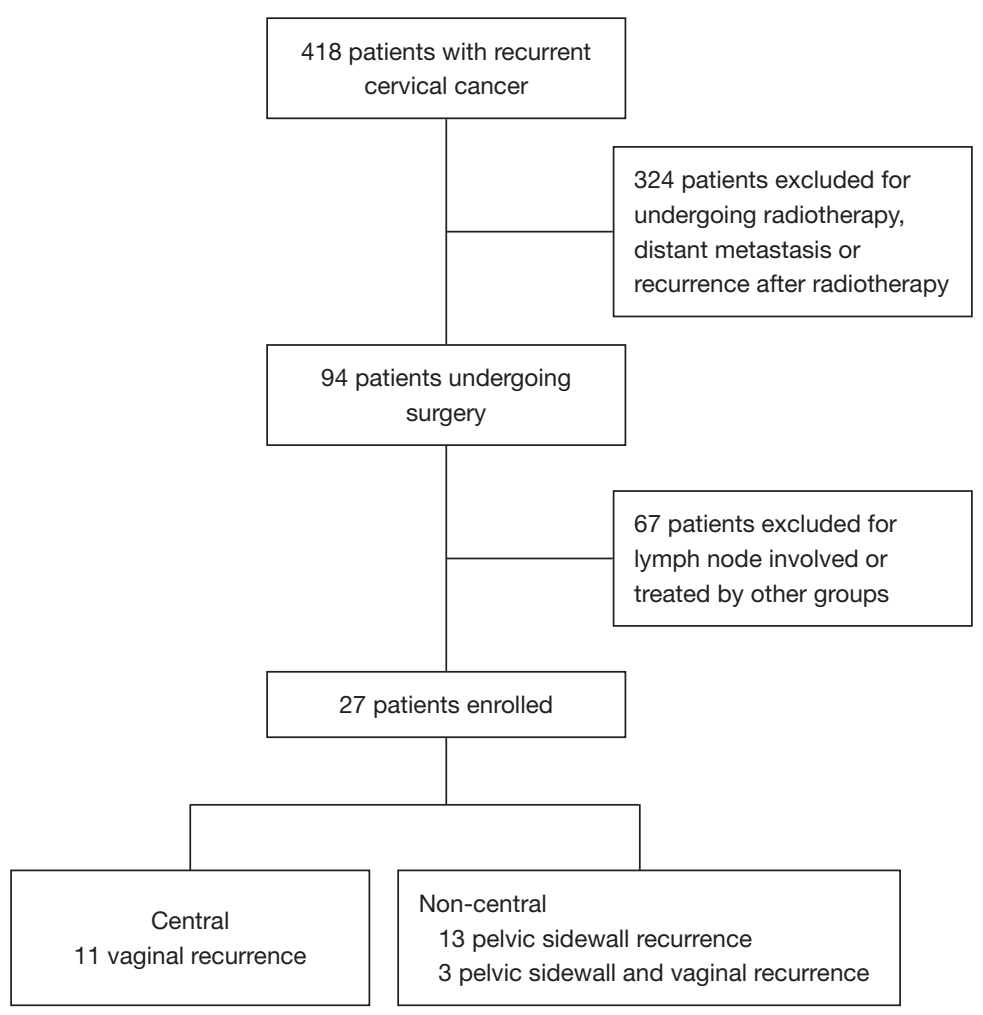

Figure 1 STROBE flow chart.

tumor. The boost volume was defined by the surgeon and radiation oncologist together, based on pelvic examination, imaging data and operation information with or without a marker of titanium clip(s). The boost dose was carried out using EBRT, vaginal brachytherapy (VBT), or both. Weekly single cisplatin chemotherapy was performed concurrently with radiotherapy with a dose of $40 \mathrm{mg} / \mathrm{m}^{2}$.

\section{Statistical analysis}

OS was defined as the time from diagnosis of recurrence to mortality, and mortality was scored as events. The occurrence of any new lesion confirmed by pathology or imaging was defined as disease progression. Progressionfree survival (PFS) was defined as the time from diagnosis of recurrence to disease progression, and mortality and disease progression were scored as events. In-field PFS (IFPFS) was defined as the time from diagnosis of recurrence to progression in the radiation field, and in-field disease progression were scored as events. Cumulative rates of OS and PFS were calculated using the Kaplan-Meier method with patients censored at the last follow-up. Log-rank tests were used to evaluate potential differences between the groups. Common Terminology Criteria for Adverse Events (v5.0) was used for the grading of complications. Statistical analyses were performed using the Statistical Package for the Social Sciences version 19 (IBM Corp, release 2010; IBM SPSS Statistics for Windows, Version 19.0; Armonk, NY, IBM Corp).

\section{Results}

\section{Patient characteristics}

This study identified 418 patients with recurrent cervical cancer from May 1998 to November 2016 in Zhejiang Cancer Hospital. Moreover, the study enrolled 27 patients with a median age of 50 years (32-69 years). Of all patients, 24 underwent radical surgery and three with stage IA1 cervical cancer underwent total hysterectomy as initial treatment (Figure 1). The median follow-up time after recurrence is 64 months (range, 5-110 months). Of the patients, 11, 13 and three had vaginal recurrence (central), pelvic sidewall recurrence, and both vaginal and pelvic sidewall recurrences (noncentral), respectively. The median time from initial surgery to the first recurrence was 
Table 1 Patient characteristics

\begin{tabular}{|c|c|c|}
\hline Characteristics & $\mathrm{N}$ & $\%$ \\
\hline \multicolumn{3}{|l|}{ Age at recurrence } \\
\hline$<50$ years & 13 & 48.1 \\
\hline$\geq 50$ years & 14 & 51.9 \\
\hline \multicolumn{3}{|l|}{ Initial FIGO stage } \\
\hline IA1 & 3 & 11.1 \\
\hline IB1 & 19 & 70.4 \\
\hline IB2 & 1 & 3.7 \\
\hline IIA1 & 3 & 11.1 \\
\hline IIA2 & 1 & 3.7 \\
\hline \multicolumn{3}{|l|}{ Primary surgical approach } \\
\hline Laparoscopic & 7 & 25.9 \\
\hline Abdominal & 14 & 51.9 \\
\hline $\mathrm{NK}^{\dagger}$ & 6 & 22.2 \\
\hline \multicolumn{3}{|l|}{ Pathology } \\
\hline Squamous cell carcinoma & 24 & 88.9 \\
\hline Adenocarcinoma & 3 & 11.1 \\
\hline \multicolumn{3}{|l|}{ Recurrence pattern and stage } \\
\hline Central (vagina) & 11 & 40.7 \\
\hline IA1 & 2 & \\
\hline IB1 & 7 & \\
\hline IB2 & 1 & \\
\hline IIA1 & 1 & \\
\hline Noncentral & 16 & 59.3 \\
\hline Pelvic sidewall & 13 & 48.1 \\
\hline $\mid \mathrm{A} 1$ & 1 & \\
\hline IB1 & 10 & \\
\hline$\| \mathrm{A} 1$ & 2 & \\
\hline Both & 3 & 11.1 \\
\hline IB1 & 2 & \\
\hline IIA2 & 1 & \\
\hline
\end{tabular}

${ }^{\dagger}$, not known. FIGO, The International Federation of Gynecology and Obstetrics 2009 staging system.
11 months (range, 3-116 months). Table 1 lists the patients' characteristics.

\section{Treatment}

All patients underwent surgery with a median intraoperative hemorrhage volume of $400 \mathrm{~mL}$ (range, $100-850 \mathrm{~mL}$ ). Nineteen patients underwent ureteral stent placement, nine of whom underwent partial cystectomy/ ureterectomy. In addition, 10 patients underwent partial bowel resection, including four small bowel resections, one sigmoid resection and five rectal resections. The goal of the surgery was to resect the tumor as completely as possible. However, four out of the 27 patients had a small residual tumor surrounding the vessels after surgery. No patient underwent ileal conduit or percutaneous cystostomy. Moreover, there was no significant statistical difference in intraoperative hemorrhage, bowel resection, partial cystectomy/ureterectomy, number of ureteral stents, and residual tumor between central and noncentral recurrences.

All patients underwent postoperative radiotherapy with a median equivalent dose in 2 Gy fractions (EQD2) of 45.1 Gy (range, 44.3-47.8 Gy). Weekly image-guided intensity-modulated radiation therapy was used for EBRT. In addition, 26 patients underwent pelvic radiotherapy and one underwent pelvic, whole vagina and inguinal radiotherapy. The median EQD2 dose for the tumor bed/ residual tumor was 51 Gy (range, 44.3-73.4 Gy), including 18 patients with a boost dose up to 50-73.4 Gy by EBRT or VBT.

Twenty-six patients underwent concurrent chemotherapy with single cisplatin for two to five cycles. One patient underwent chemotherapy (cisplatin/paclitaxel for four cycles) before radiotherapy due to the presence of a ureteral intestinal fistula after surgery.

Table 2 lists treatment details. Eight patients had multiple recurrent lesions after the operation. The initial treatment of four patients was laparoscopic surgery and the other four were laparotomy. The locations of multiple recurrences were in the mesentery and abdominal wall. Table 3 provides postoperative pathological details. 
Table 2 Treatment details

\begin{tabular}{|c|c|c|c|c|}
\hline Treatment & Total $(n=27)$ & Central $(n=11)$ & Noncentral $(n=16)$ & $P$ value \\
\hline Median & 400 & 400 & 400 & \\
\hline Range & $100-850$ & $100-850$ & $200-800$ & \\
\hline Ureteral stent, n (\%) & & & & 0.183 \\
\hline Unilateral & $10(37.0)$ & $2(18.2)$ & $8(50.0)$ & \\
\hline Bilateral & $9(33.3)$ & $4(36.4)$ & $5(31.3)$ & \\
\hline Bowel resection, n (\%) & $10(33.3)$ & $3(27.3)$ & $6(37.5)$ & 0.384 \\
\hline Partial small bowel resection & $4(14.8)$ & $1(9.1)$ & $3(18.8)$ & \\
\hline Cystectomy/ureterectomy & $9(33.3)$ & $3(27.3)$ & $6(37.5)$ & 0.580 \\
\hline Residual tumor, n (\%) & $4(14.8)$ & $2(18.2)$ & $2(12.5)$ & 0.683 \\
\hline EQD2 dose for tumor bed/residual tumor (Gy) & & & & 0.257 \\
\hline Median & 51 & 52 & 49.8 & \\
\hline Range & $44.3-73.4$ & $44.3-72.6$ & $44.3-73.4$ & \\
\hline Time from surgery to radiotherapy (days) & & & & 0.376 \\
\hline Median & 27 & 28 & 27 & \\
\hline Range & $17-186$ & $18-30$ & $17-186$ & \\
\hline
\end{tabular}

EQD2, biologically equivalent dose in 2 Gy fractions.

\section{OS}

The 5 -year OS rate of the entire group was $71.7 \%$ (Figure 2A). For central and noncentral recurrence, the 5 -year OS rates were $77.1 \%$ and $65.7 \%$, respectively. No statistically significant difference was noted regarding the recurrent pattern (central vs. noncentral, $\mathrm{P}=0.246$, Figure $2 B$ ) or residual tumor $(\mathrm{P}=0.881$, Figure $2 C)$.

By the end of the study follow-up, 12 and 6 patients had survived $>4$ and $>6$ years following treatment of the first recurrence, respectively.

\section{PFS}

The 5-year PFS rate of the entire group was $52.8 \%$ (Figure $3 A$ ). For central and noncentral recurrence, the 5 -year PFS rates were $81.8 \%$ and $34.4 \%$, respectively. There was a statistically significant difference regarding the recurrent pattern (central vs. noncentral, $\mathrm{P}=0.047$,
Figure 3B). However, there was no statistically significant difference regarding the dose for tumor bed/residual tumor ( $\geq 60$ vs. $<60$ Gy, $\mathrm{P}=0.730$, Figure $3 C$ ).

The 5-year IF-PFS rate of the entire group was $72.8 \%$ (Figure 3D). For central and noncentral recurrences, the 5 -year IF-PFS rates were $90.9 \%$ and $60.2 \%$, respectively. There was no statistically significant difference regarding the recurrent pattern (central or noncentral, $\mathrm{P}=0.128$, Figure $3 E$ ) or tumor bed/residual tumor dose ( $\geq 60$ vs. $<60 \mathrm{~Gy}, \mathrm{P}=0.157$, Figure $3 F)$. However, all five patients receiving $\geq 60$ Gy for tumor bed/residual tumor survived without in-field disease.

By the end of the study follow-up, 10 and 5 patients had survived $>4$ and $>6$ years without disease progression following treatment of the first recurrence, respectively.

\section{Patterns of progression}

Twelve (44.4\%) patients experienced disease progression 
after treatment of the first recurrence. The median time from the first to the second recurrence was 5 months (2-34 months). Ten (83.3\%) recurrences occurred within 12 months. There were six in-field failures, five out-field failures including in the para-aortic lymph node $(\mathrm{n}=1)$,

Table 3 Pathological characteristics after surgery for recurrent cervical cancer patients

\begin{tabular}{ll}
\hline Characteristics & Value \\
\hline Tumor diameter (cm) & Median, 5; range, 2-10 \\
Differentiation (n) & 0 \\
Grade 1 & 9 \\
Grade 2 & 16 \\
Grade 3 & 2 \\
NK & 13 \\
Lymphovascular space invasion (n) & 10 \\
Perineural invasion (n) & \\
Lesion (n) & 19 \\
One & 3 \\
Laparoscopy as initial treatment & 16 \\
Laparotomy as initial treatment & 8 \\
Two or more & 4 \\
Laparoscopy as initial treatment & 4 \\
Laparotomy as initial treatment & \\
\hline NK, not known. & \\
\hline
\end{tabular}

NK, not known. urethra ( $\mathrm{n}=1)$, lung $(\mathrm{n}=1)$, liver $(\mathrm{n}=1)$ and inguinal lymph node $(\mathrm{n}=1)$, and one in both in- and out-field failures (abdominal wall). Of the 12 patients, 3 (25\%) and 9 (75\%) had pathological adenocarcinoma (all adenocarcinoma patients in this study) and noncentral recurrence, respectively.

Where possible, all 12 patients underwent salvage chemotherapy with regional therapy (surgery, radiotherapy and ablation therapy). Their median OS was 27 months (5-72 months) after disease progression. Three patients, including patients who had pelvic recurrence $(\mathrm{n}=1)$, paraaortic metastasis $(\mathrm{n}=1)$ and lung metastasis $(\mathrm{n}=1)$, survived by the end of the study follow-up.

\section{Complications}

Of the 27 patients, three with noncentral recurrence had experienced grade $\geq 3$ complications associated with surgery, including one vesicovaginal fistula, one vesicovaginal and intestinal fistula, and one vesicovaginal fistula and intestinal obstruction. All these complications were successfully treated with surgery or conservative treatment. Six patients experienced repeated urinary tract infections after ureteral stent(s) placement, which were resolved by removing the ureteral stent(s).

No grade $\geq 3$ complications in the intestine, urinary system, and thrombocytes occurred during radiotherapy and chemotherapy. Grade $\geq 3$ hematotoxicity, for example, neutropenia and anemia, were observed in $7(25.9 \%)$ and $10(37.0 \%)$ patients, respectively. However, these complications associated with radiotherapy
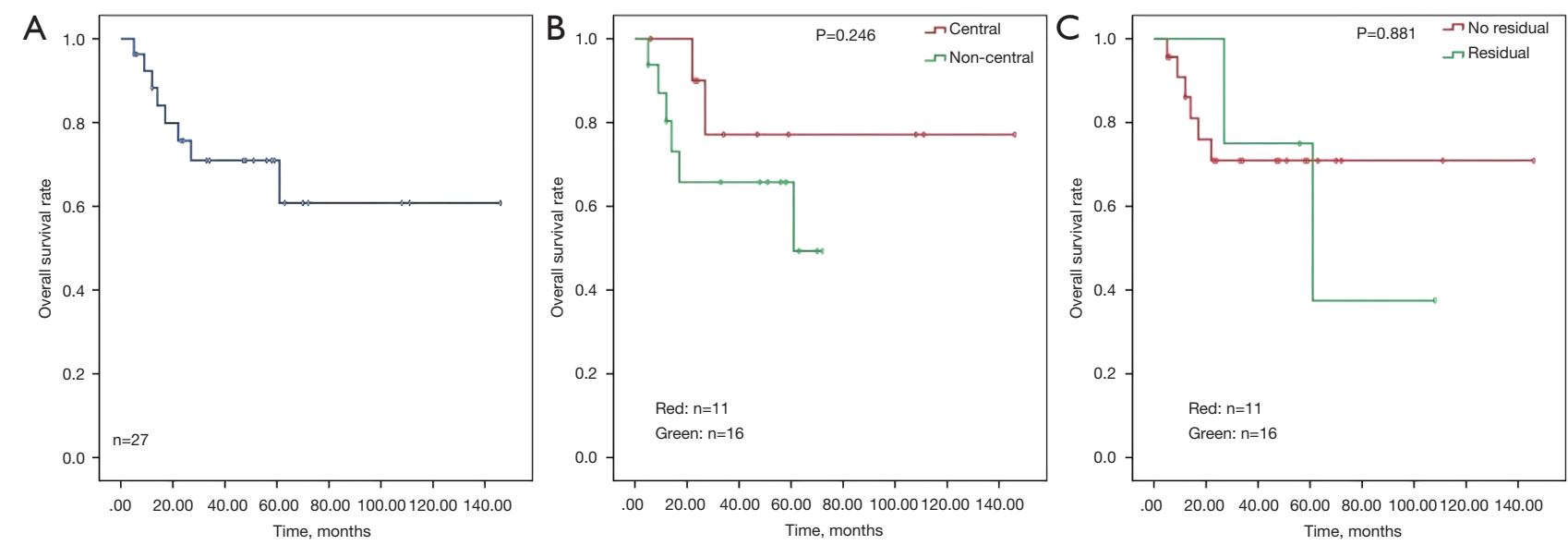

Figure 2 OS curves. (A) OS for the whole group. (B) OS for central (red) and noncentral (green) recurrences, respectively. (C) OS for nonresidual (red) and residual (green) tumors, respectively. OS, overall survival. 

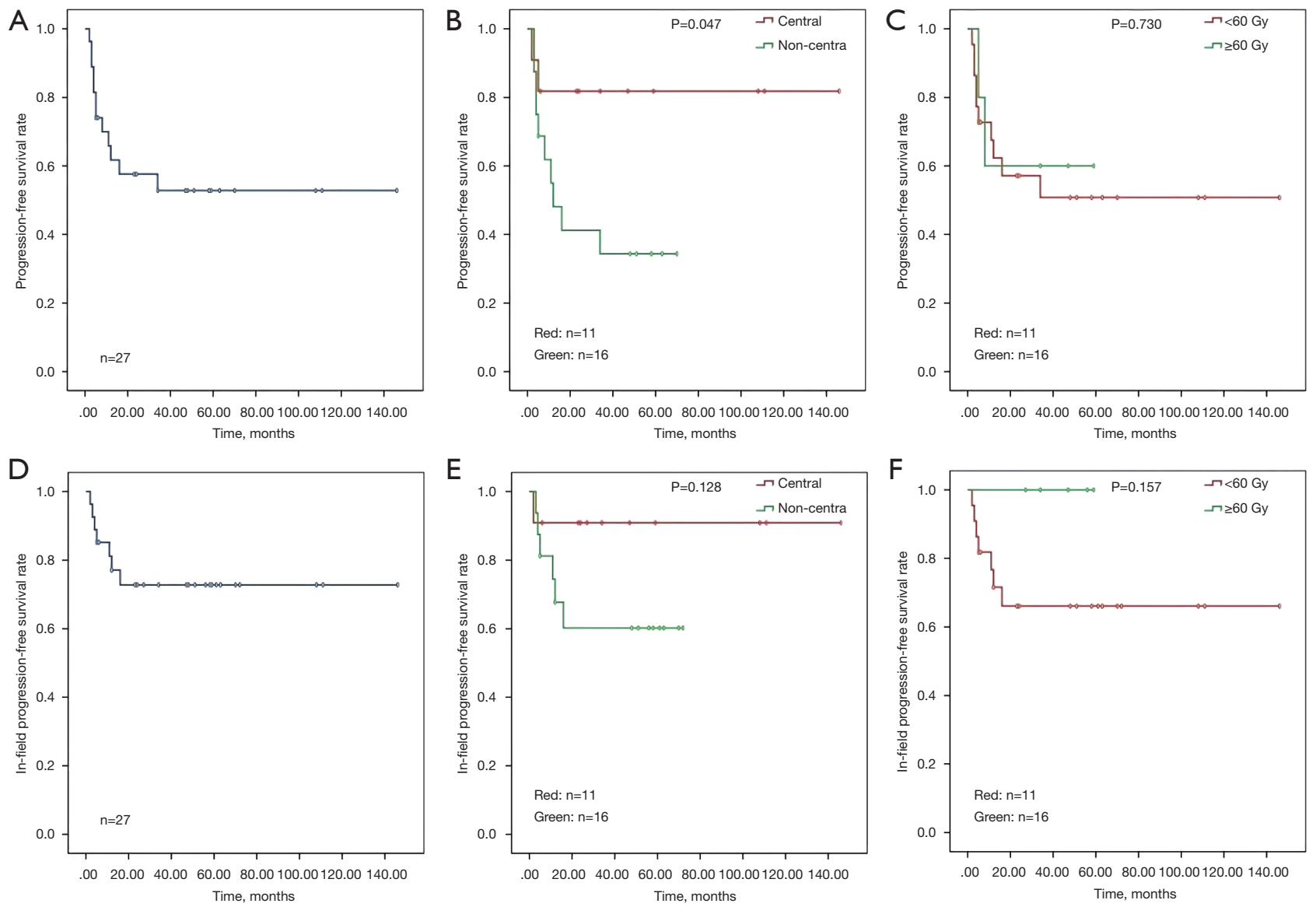

Figure 3 PFS curves. (A) PFS for the whole group. (B) PFS for central (red) and noncentral (green) recurrences, respectively. (C) PFS for the dose of tumor bed/residual tumor (red <60 Gy, green $\geq 60 \mathrm{~Gy}$ ). (D) IF-PFS for the whole group. (E) IF-PFS for central (red) and noncentral (green) recurrences, respectively. (F) IF-PFS for the dose of tumor bed/residual tumor (red <60 Gy, green $\geq 60$ Gy). PFS, progression-free survival; IF-PFS, In-field PFS.

Table 4 Details of grade $\geq 3$ complications

\begin{tabular}{lcc}
\hline Complications & $\mathrm{N}$ & $\%$ \\
\hline Surgery & 1 & 3.7 \\
Vesicovaginal fistula & 1 & 3.7 \\
Intestinal fistula & & \\
Radiochemotherapy & 2 & 7.4 \\
Vesicovaginal fistula & 1 & 3.7 \\
Intestinal obstruction & 7 & 25.9 \\
Neutropenia & 0 & 0 \\
Thrombocytopenia & 10 & 37.0 \\
Anemia & & \\
\hline
\end{tabular}

or radiochemotherapy had no effects on the treatment progress. Table 4 shows details of complications.

\section{Discussion}

Treatment of recurrent cervical cancer remains a challenge, especially in noncentral recurrence. According to the NCCN guideline for cervical cancer, local recurrence of cervical cancer without previous radiotherapy may be considered for surgery. However, the role of surgery following concurrent radiochemotherapy has not been well-studied, with no high-quality evidence cited by the guideline. This study focused on the role of local surgery 
and subsequent radiochemotherapy for recurrent cervical cancer without previous radiotherapy, whether central or noncentral recurrences. The 5-year OS rate in this study were $71.7 \%, 77.1 \%$ and $65.7 \%$ in all patients, in patients with central recurrence and patients with noncentral recurrence, respectively.

For central recurrence, the 5-year OS rate of this study was consistent with that observed in the previous studies $(5-10,12)$. However, the 5 -year OS rate of $65.7 \%$ for noncentral recurrence was higher than that in the previous studies, mainly using radiotherapy alone or with chemotherapy. Ijaz et al. (6) reported a 5-year OS rate of $18 \%$ in a group of 11 patients with central recurrence and pelvic wall extension treated by EBRT with or without intracavitary or ${ }^{198} \mathrm{AU}$ seeds BT. Haasbeek et al. (5) reported that 35 patients with recurrent cervical cancer underwent salvage radiotherapy. Of the 35 patients, 20 had pelvic sidewall recurrence and underwent radiotherapy with a median dose of 60.8 Gy (50-80 Gy) using different radiation technologies for over 30 years. The 5-year OS rate for the 20 patients was $28 \%$. In 2012, Qiu et al. (7) reported a 5 -year OS rate of $41.82 \%$ in 22 patients with recurrent pelvic cervical cancer (not central or vaginal) in their study, which included 106 recurrent cervical cancer patients. This 5-year OS rate was higher than the previous studies. However, they did not report the details of the treatment for the 22 patients. Kim et al. (13) conducted a study using modern radiotherapy (three-dimensional conformal radiotherapy or intensity-modulated radiotherapy) to treat recurrent or metastatic cervical cancer. In their study, the 5-year OS rate was up to $66 \%$ for all patients. However, only two groups of patients had local recurrence (i.e., cervix or vaginal vault and pelvic LN). Moreover, they did not report the number of patients with pelvic sidewall recurrence. Therefore, this is the first study that confirms that long-term survival for patients with recurrent cervical cancer, especially pelvic sidewall recurrence, could be achieved by combining surgery and concurrent radiochemotherapy.

Traditionally, noncentral recurrence was considered with worse survival compared to central recurrence. However, the 5-year OS rate of noncentral recurrence was much better than in previous studies. This was possible mainly because local surgery played a key role in treating patients with pelvic sidewall recurrence. Reaching the radiation dose of $\geq 70 \mathrm{~Gy}$, a theoretically lethal dose for a $2-\mathrm{cm}$ tumor, is generally difficult for the pelvic sidewall recurrence. However, a relatively low radiation dose can achieve curative treatment after a tumor has been resected or reduced as completely as possible by surgery. A boosted dose delivered in the tumor bed for patients with residual tumor or without safe margin may play a role in tumor local control after surgery. Although there was no statistical difference following the tumor bed/residual tumor dose, all patients $(n=5)$ with a tumor bed/residual tumor dose of $\geq 60$ Gy survived without in-field failure. In the early stages of the current study, the pelvic sidewall was not boosted to $\geq 60 \mathrm{~Gy}$, which may explain the statistically shorter PFS of the pelvic wall recurrence than that of the central recurrence. The dose of tumor bed/residual tumor was recently boosted to at least 60 Gy for every patient. However, only some of these patients were included in the current study due to the short follow-up period.

Before this study, Höckel et al. $(14,15)$ described in 1991 a treatment, named combined operative and radiotherapeutic treatment (CORT), for recurrent tumors infiltrating in the unilateral pelvic wall. The main operative procedures included maximum tumor debulking and exenteration of infiltrated organs; implantation of tubes on the residual tumor/tumor bed for BT; pelvic wall plasty with muscle, musculocutaneous and omentum flaps; and organ reconstruction (14). BT was performed after surgery for tumor bed/residual tumor irradiation. This treatment was mainly designed for recurrent patients who underwent previous pelvic radiotherapy. In 1996, 48 patients reportedly had 5 -year survival and severe complication rates of $44 \%$ and $33 \%$, respectively, after treatment (16). This result showed that surgery combined with radiotherapy was effective for recurrent patients. However, few studies focused on surgery combined with radiotherapy for patients with recurrent cervical cancer partly because of the difficulty in surgery and the presence of a high severe complication rate. In the current study, the difficulty in performing the surgery was not as high as that for CORT because the patients did not undergo radiotherapy before, and the safety margin was not indispensable. Additionally, modern EBRT, instead of BT, could deliver an adequate radiation dose to the pelvic sidewall after surgery. Thus, implantation of BT tubes was not necessary to reduce the surgical difficulty and postoperative complications. Therefore, performing surgery combined with radiotherapy for recurrent patients was feasible in the current study.

The recurrence after radical laparoscopic hysterectomy and lymph node dissection, a new situation, would confuse the radiation oncologists. Pelvic recurrence generally involves a single lesion in the era of open surgery except for lymph node recurrence. However, multilesion 
recurrence after laparoscopic surgery is 3.5 times higher than open surgery $(17,18)$. Hence, radiation oncologists have to search for possible missing lesions carefully. In the current study, seven patients were noted with recurrence after laparoscopic surgery, and four of them experienced multilesion recurrence. Preoperative images did not detect all recurrent lesions compared to the findings during the operation. Therefore, surgery for patients with recurrence, especially for patients undergoing previous laparoscopic radical surgery, is a treatment and an assessment on how to perform effective radiotherapy according to surgical and pathologic factors.

Of the three surviving patients with a second recurrence, two survived without disease progression after salvage treatment. One of them experienced recurrence in the abdominal wall and partial right iliopsoas, partly involved in the previous radiation volume. The patient underwent surgery and radiotherapy again. Recurrent tumors were completely resected, and a 40-Gy radiation dose was carefully delivered to the tumor beds. The patient survived without disease progression and severe complications for 33 months. Another patient experienced lung metastasis and underwent surgery and chemotherapy for six cycles. At the end of the study follow-up, the patient had survived without disease progression and severe complications for 51 months. The successful salvage treatment confirmed the role of surgery in the treatment of recurrent patients.

Several complications were notable in the current study. Vesicovaginal fistula $(n=3)$, intestinal fistula $(n=1)$ and intestinal obstruction $(\mathrm{n}=1)$ were observed. One patient experienced a vesicovaginal fistula followed by an intestinal fistula. However, severe complications were much lower than CORT's. These complications resolved after treatment. The patient who experienced a vesicovaginal fistula followed by an intestinal fistula delayed the start of radiotherapy for 6 months and one patient with a vesicovaginal fistula experienced a 2 -month radiotherapy interruption. However, the delay or interruption of radiotherapy does not affect survival. The two patients still survived without disease progression by the end of the study follow-up. Six patients experienced repeated urinary tract infection during postoperative radiotherapy mainly because ureteral stent, a foreign matter in the body, may become a place for bacterial cultivation. These patients with repeated urinary tract infections were cured after removing the ureteral stent(s). Thus, integration of local surgery and modern radiotherapy was well tolerable for recurrent cervical cancer patients.
This study has several limitations. First, patients from the same surgery and radiotherapy team were selected, resulting in a small sample size to ensure the homogeneity of surgery and radiotherapy. Second, as a retrospective study, some protocols changed (e.g., the radiation dose of the tumor bed). Third, no control group of patients receiving concurrent radiochemotherapy was noted; therefore, the data were compared with previous studies.

\section{Conclusions}

In conclusion, the combination of local surgery and radiochemotherapy is a feasible and effective treatment with acceptable complications for locally recurrent cervical cancer, improving pelvic sidewall recurrence survival. In the future, prospective clinical trials could confirm the role of surgery and determine the details of surgery and radiotherapy.

\section{Acknowledgments}

Funding: This work was supported by the Natural Science Foundation of Zhejiang Province (LY17H160037), Zhejiang Medical and Health Science and Technology Project (2018KY299) and Zhejiang Traditional Chinese Medicine Science and Technology Plan (2015ZB018).

\section{Footnote}

Reporting Checklist: The authors have completed the STROBE reporting checklist. Available at https://dx.doi. org/10.21037/tcr-21-1163

Data Sharing Statement: Available at https://dx.doi. org/10.21037/tcr-21-1163

Peer Review File: Available at https://dx.doi.org/10.21037/ tcr-21-1163

Conflicts of Interest: All authors have completed the ICMJE uniform disclosure form (available at https://dx.doi. org/10.21037/tcr-21-1163). The authors have no conflicts of interest to declare.

Ethical Statement: The authors are accountable for all aspects of the work in ensuring that questions related to the accuracy or integrity of any part of the work are appropriately investigated and resolved. The study was 
conducted following the Declaration of Helsinki (as 2013 revision). The study was approved by the Ethics Committee of Zhejiang Cancer Hospital (No. 2015-01-99) and individual consent for this retrospective analysis was waived.

Open Access Statement: This is an Open Access article distributed in accordance with the Creative Commons Attribution-NonCommercial-NoDerivs 4.0 International License (CC BY-NC-ND 4.0), which permits the noncommercial replication and distribution of the article with the strict proviso that no changes or edits are made and the original work is properly cited (including links to both the formal publication through the relevant DOI and the license). See: https://creativecommons.org/licenses/by-nc-nd/4.0/.

\section{References}

1. Bray F, Ferlay J, Soerjomataram I, et al. Global cancer statistics 2018: GLOBOCAN estimates of incidence and mortality worldwide for 36 cancers in 185 countries. CA Cancer J Clin 2018;68:394-424.

2. Quinn MA, Benedet JL, Odicino F, et al. Carcinoma of the Cervix Uteri. Int J Gynaecol Obstet 2006;95 Suppl 1:S43103.

3. Pötter R, Tanderup K, Schmid MP, et al. MRI-guided adaptive brachytherapy in locally advanced cervical cancer (EMBRACE-I): a multicentre prospective cohort study. Lancet Oncol 2021;22:538-47.

4. Kim YJ, Kang HC, Kim YS. Impact of intracavitary brachytherapy technique (2D versus $3 \mathrm{D}$ ) on outcomes of cervical cancer: a systematic review and meta-analysis. Strahlenther Onkol 2020;196:973-82.

5. Haasbeek CJ, Uitterhoeve AL, van der Velden J, et al. Long-term results of salvage radiotherapy for the treatment of recurrent cervical carcinoma after prior surgery. Radiother Oncol 2008;89:197-204.

6. Ijaz T, Eifel PJ, Burke T, et al. Radiation therapy of pelvic recurrence after radical hysterectomy for cervical carcinoma. Gynecol Oncol 1998;70:241-6.

7. Qiu JT, Abdullah NA, Chou HH, et al. Outcomes and prognosis of patients with recurrent cervical cancer after

Cite this article as: Zhang X, Chen Z, Chen J, Wang J, Wang Y, Zhu J. Surgery followed by concurrent radiochemotherapy as treatment for patients with locally recurrent cervical cancer. Transl Cancer Res 2021;10(10):4365-4374. doi: 10.21037/tcr21-1163 radical hysterectomy. Gynecol Oncol 2012;127:472-7.

8. Hille A, Weiss E, Hess CF. Therapeutic outcome and prognostic factors in the radiotherapy of recurrences of cervical carcinoma following surgery. Strahlenther Onkol 2003;179:742-7.

9. Jain P, Hunter RD, Livsey JE, et al. Salvaging locoregional recurrence with radiotherapy after surgery in early cervical cancer. Clin Oncol (R Coll Radiol) 2007;19:763-8.

10. Wang CJ, Lai CH, Huang HJ, et al. Recurrent cervical carcinoma after primary radical surgery. Am J Obstet Gynecol 1999;181:518-24.

11. Kim SW, Chun M, Ryu HS, et al. Salvage radiotherapy with or without concurrent chemotherapy for pelvic recurrence after hysterectomy alone for early-stage uterine cervical cancer. Strahlenther Onkol 2017;193:534-42.

12. Hong JH, Tsai CS, Lai CH, et al. Recurrent squamous cell carcinoma of cervix after definitive radiotherapy. Int J Radiat Oncol Biol Phys 2004;60:249-57.

13. Kim HJ, Chang JS, Koom WS, et al. Radiotherapy is a safe and effective salvage treatment for recurrent cervical cancer. Gynecol Oncol 2018;151:208-14.

14. Höckel M, Knapstein PG. The combined operative and radiotherapeutic treatment (CORT) of recurrent tumors infiltrating the pelvic wall: first experience with 18 patients. Gynecol Oncol 1992;46:20-8.

15. Höckel M, Knapstein PG, Kutzner J. A novel combined operative and radiotherapeutic treatment approach for recurrent gynecologic malignant lesions infiltrating the pelvic wall. Surg Gynecol Obstet 1991;173:297-302.

16. Höckel M, Sclenger K, Hamm H, et al. Five-year experience with combined operative and radiotherapeutic treatment of recurrent gynecologic tumors infiltrating the pelvic wall. Cancer 1996;77:1918-33.

17. Ramirez PT, Frumovitz M, Pareja R, et al. Minimally Invasive versus Abdominal Radical Hysterectomy for Cervical Cancer. N Engl J Med 2018;379:1895-904.

18. Bogani G, Ghezzi F, Chiva L, et al. Patterns of recurrence after laparoscopic versus open abdominal radical hysterectomy in patients with cervical cancer: a propensitymatched analysis. Int J Gynecol Cancer 2020;30:987-92 . 